REVISTA MATEMÁTICA de la

Universidad Complutense de Madrid

Volumen 6, número 1; 1993.

http://dx.doi.org/10.5209/rev_REMA.1993.v6.n1.17841

\title{
The Irreducible Components of the Nilpotent Associative Algebras
}

\author{
ABDENACER MAKHLOUF
}

\begin{abstract}
The aim of this work is to describe the irreducible components of the nilpotent complex associative algebras varieties of dimension 2 to 5 ; and to give a lower bound of the number of these components in any dimension.
\end{abstract}

\section{INTRODUCTION}

Let $\mathcal{N}^{n}$ be the variety of the $n$-dimensional nilpotent complex associative algebras (without unity). The direct product $\mathcal{N}^{n} \mathrm{x} \mathbb{C}$ is a Zariskiclosed subset of $\mathrm{Alg}_{n+1}$ which is the variety of $(n+1)$-dimensional unitary complex associative algebras.

An associative algebra $A$ is nilpotent if the descending chain of ideals $A \supset A^{2} \supset \ldots$ terminates. If $A^{s} \neq\{0\}$ and $A^{s+1}=\{0\}$ then $s$ is the nilindex of $A$.

The group $\mathrm{GL}(\mathrm{n}, \mathbb{C})$ acts on $\mathcal{K}^{n}$. Since the classification, up to isomorphism, of $\mathrm{Alg}_{n}$ is known until $\mathrm{n}=5$, one can deduce the classification of $\mathfrak{N}^{n}$ until $\mathrm{n}=4$. One can also get the irreducible components from the deformation diagrams for these dimensions.

1991 Mathematics Subject Classification: 16P10, 16R10, 16N40, 16D70.

Editorial Complutense. Madrid, 1993. 
In this paper, we describe the irreducible components of $\mathcal{N}^{n}$ for $n=1$ to 5 using the nilindex invariant. This approach follows the remark that the nilindex does not decrease by perturbation. Then, by perturbation, it is easy to see if a given algebra of fixed nilindex defines an irreducible component or can be perturbed in an algebra of larger nilindex.

Moreover, in $\mathcal{N}^{\pi}$, we present for each nilindex different from one, a family of algebras which could not be perturbed in algebras of larger nilindex. Therefore, we deduce a lower bound of the number of the irreducible components of $\mathcal{N}^{\mathrm{n}}$.

Finally, using the study of $\mathcal{N}^{5}$, we show that in $\mathrm{Alg}_{6}$ there are no rigid nilpotent algebras if a nilpotent algebra of $\mathrm{Alg}_{6}$ is identified with an element of $\mathbb{C} \times \mathfrak{N}^{5}$.

\section{NILINDEX AND PERTURBATION}

In this work, the notion of perturbation related to the Nonstandard framework ([G],[G-M]), is used instead of the deformation notion.

Let $A=\left(\mathbb{C}^{n}, \mu_{0}\right)$ be a standard algebra of $\mathcal{N}^{n}$. It is defined by its structure constants $\left(C_{\mathrm{ij}}^{\mathrm{k}}\right)_{1 \leq \mathrm{i}, \mathrm{j}, \mathrm{k} \leq \mathrm{n}}$ in a fixed standard basis $\mathcal{B}$.

Definition. $A$ perturbation of $A$ is an algebra $A^{\prime}=\left(\mathbb{C}^{n}, \mu\right)$ of $\mathcal{X} \mathbb{C}^{n}$ such that $\mu(X, Y)-\mu_{0}(X, Y)$ is infinitesimal for any standard elements $X, Y$ in $\mathbb{C}^{n}$.

If this is the case we write $\mu \propto \mu_{0}$.

The structure constants of $A^{\prime}$ and $A$, in a standard fixed basis of $\mathbb{C}^{n}$ are infinitely closed.

Definition. The algebra $A_{t}=\left(\mathbb{C}^{\mathrm{n}}, \mu_{t}\right)$ of $\mathfrak{N}$ is a contraction of $A=\left(\mathbb{C}^{n}, \mu_{0}\right)$ if there exist a perturbation $A^{\prime}=\left(\mathbb{C}^{n}, \mu\right)$ of $A_{1}$ such that $A^{\prime}$ is isomorphic to $A$. 


\section{Remarks.}

$\left.1^{9}\right)$ A contraction of a standard algebra is also called a specialisation.

$\left.2^{2}\right)$ Let $A_{1}=\left(\mathbb{C}^{n}, \mu_{1}\right)$ in $\mathcal{N}^{n}$ be a contraction of $A=\left(\mathbb{C}^{n}, \mu_{0}\right)$, then $A_{1}=\left(\mathbb{C}^{n}, \mu_{1}\right)$ is in the closure of the orbit of $A=\left(\mathbb{C}^{n}, \mu_{0}\right)$ corresponding to the natural action of GL(n, C) on $\mathcal{X N}^{n}$.

Perturbation of the nilindex. Let $A=\left(\mathbb{C}^{n}, \mu_{0}\right)$ be a standard algebra of $\mathcal{X} C^{n}$ and $s$ the nilindex of $A$.

$$
\mathrm{s}=\min \left\{\mathrm{p}: \mathrm{X}^{\mathrm{p}+1}=0 \quad \forall \mathrm{X} \in \mathrm{A}\right\}
$$

Lemma. If $A^{\prime}=\left(\mathbb{C}^{n}, \mu\right)$ is a perturbation of $A$ then the nilindex of $A^{\prime}$ is larger or equal to $s$.

Proof. We have $A^{s} \neq\{0\}$. Since $A$ is standard then $\left(A^{\prime}\right)^{s} \neq\{0\}$.

This statement is fundamental. If we cannot perturb an algebra in an algebra of larger nilindex, namely if this algebra is not a contraction of an algebra of larger nilindex, then this algebra does not belong to a component containing an algebra of larger nilindex. It will be used in the following cases.

\section{LOWER BOUND OF THE NUMBER OF THE IRREDUCIBLE COMPONENTS}

As an illustration of the previous method in $\mathcal{N}^{\mathrm{n}}, \mathrm{n}>2$, we present for each value $s$ of the nilindex, $1<s<n$, an algebra $A_{s}$ such that every perturbation has the same nilindex. Throughout this paper, the products not written are null. 
Definition. The algebra $A_{s}^{n}$ of $\mathcal{N}^{\mathrm{n}}$ and nilindex $s$ is defined by the following law: Assume that $X$ satisfies $X^{s} \neq 0$. Let $\left(X, X^{2}, \ldots, U_{I}, \ldots, U_{n-s}\right)$ be the basis of $A_{s}^{n}$. Then

$$
\begin{aligned}
& X^{i} \cdot X^{j}=X^{i+j} \quad \text { if } i+j \leq s \\
& X \cdot U_{i}=X^{s} \quad i=1, \ldots, n-s \\
& U_{i} \cdot U_{j}=\gamma_{i}^{j} X^{s} \quad \gamma_{i}^{j} \in \mathbb{C} \quad i, j=1, \ldots, n-s .
\end{aligned}
$$

Proposition. Every perturbation of $A_{s}^{n}$ has nilindex $s$.

Proof. Let $A^{\prime}$ be a perturbation of $A_{s}^{n}$. Assume that $A^{s+1} \neq 0$. Then we have:

$$
X^{s+1}=\varepsilon_{1} X+\varepsilon_{2} X^{2}+\ldots+\varepsilon_{s} X^{s}+\varepsilon_{s+1} U_{1}+\ldots+\varepsilon_{n} U_{n-s}, \quad \varepsilon_{i} \propto 0 \quad i=1, \ldots, n
$$

We have $\varepsilon_{s+2}=\ldots=\varepsilon_{n}=0$ by the isomorphism:

$$
\left\{X^{\prime}=X ; U_{1}^{\prime}=U_{1}+\left(1 / \varepsilon_{s+1}\right)\left(\varepsilon_{s+2} U_{2}+\ldots+\varepsilon_{n} U_{n-s}\right) ; U_{i}^{\prime}=U_{i} \quad i=2, \ldots, n-s\right.
$$

Therefore $\quad X^{s+2}=\varepsilon_{1} X^{2}+\varepsilon_{2} X^{3}+\ldots+\varepsilon_{s} X^{s+1}+\varepsilon_{s+1} X \cdot U_{1}$

and $X^{s+2}=\varepsilon_{1} X^{2}+\varepsilon_{2} X^{3}+\ldots+\varepsilon_{s} X^{s+1}+\varepsilon_{s+1} U_{1} \cdot X$.

The difference of the two equations implies

$$
\varepsilon_{\mathrm{s}+1}\left(X \cdot U_{1}-U_{1} \cdot X\right)=0
$$

since $X \cdot U_{1}=\phi_{1}$ and $U_{1} \cdot X=X^{s}+\phi_{2}$ where $\phi_{1}$ and $\phi_{2}$ are infinitesimal vectors. On the component $X^{s}$, we have $\varepsilon_{s+1}\left(-1+\phi_{2}^{s}-\phi_{1}^{s}\right)=0$. Then $\varepsilon_{s+1}$ $=0$.

We have the following consequence.

Theorem. \# (irreducible components of $\mathcal{N}^{\mathrm{N}}$ ) $\geq n-1$.

Proof. If the nilindex is maximal $(s=n)$, then the corresponding algebra is rigid. Therefore it determines an irreducible component. 
If $1<\mathrm{s}<\mathrm{n}$, for each value of the nilindex, following the previous proposition, there is an irreducible component.

\section{IRREDUCIBLE COMPONENTS OF $\mathfrak{N}^{2}, \mathcal{N}^{3}, \mathcal{N}^{4}$ AND $\mathfrak{N}^{5}$}

The classification and deformation diagram of $\mathrm{Alg}_{\mathrm{n}}$ is known for $\mathrm{n}=2, \ldots, 5$ (see $[\mathrm{Ga}],[\mathrm{Ma}],[\mathrm{H}]$ ). From these results one can deduce the irreducible components of $\mathcal{N}^{2}, \mathcal{N}^{3}$ and $\mathcal{N}^{4}$. This study is done here directly, whithout using the complete classifications. Hence, we determine the irreducible components of $\mathfrak{N}^{5}$. The technique is the same as above. For each value of the nilindex the study is based on the following lemma.

Lemma. Let $A$ be in $\mathcal{N}^{n}$, s the nilindex of $A$ and $X \in A$ such that $X^{s} \neq 0$. Then the Jordan blocs of the operator $L_{X}\left(L_{X}(Y)=X \cdot Y\right)$ are at most $(s+1)$ dimensional, and the subspace generated by $\left(X, X^{2}, \ldots, X^{s}\right)$ is invariant by $L_{X}$.

Proof. Since $\mathrm{L}_{\mathrm{x}^{\mathrm{s}+1}}=0$ then the Jordan blocs have at most the lenght $s+1$.

The vector $X^{s}$ is an eigenvector of $L_{X}$. Then $\left(X, \ldots, X^{s}\right)$ are in the subspace invariant by $\mathrm{L}_{\mathrm{X}}$. Suppose that it exists $\mathrm{U}$ such that $\mathrm{L}_{\mathrm{X}} \mathrm{U}=\mathrm{X}$, then the algebra is not nilpotent.

\section{VARIETY $\mathfrak{X}^{2}$.}

$\mathcal{N}^{2}$ is formed only by 2 algebras, up to isomorphism. Let $\left\{\mathrm{e}_{1}, \mathrm{e}_{2}\right\}$ be a basis of $\mathbb{C}^{2}$. There is, in $\mathcal{N}^{2}$, the following laws:

$\left.1^{a}\right) \mu_{1}^{2}\left(e_{1}, e_{1}\right)=e_{2}$.

$\left.2^{\underline{9}}\right)$ The null algebra. $\mu_{1}^{2}$.

It's easy to see that the null algebra is in the closure of the orbit of 
Proposition. The variery $\mathfrak{N}^{2}$ has one irreducible component.

VARIETY $\mathfrak{x}^{3}$

Proposition. The variety $\mathcal{N}^{3}$ has 2 irreducible components.

Proof. We have the following cases of the matrix of the operator $\mathrm{L}_{\mathrm{x}}$.
$\left.1^{9}\right)\left(\begin{array}{lll}0 & 0 & 0 \\ 1 & 0 & 0 \\ 0 & 1 & 0\end{array}\right)$
$\left.2^{2}\right)\left(\begin{array}{lll}0 & 0 & 0 \\ 1 & 0 & 0 \\ 0 & 0 & 0\end{array}\right)$
39) $\left(\begin{array}{lll}0 & 0 & 0 \\ 0 & 0 & 0 \\ 0 & 1 & 0\end{array}\right)$
$\left.4^{9}\right)\left(\begin{array}{lll}0 & 0 & 0 \\ 0 & 0 & 0 \\ 0 & 0 & 0\end{array}\right)$

$1^{\text {g) }}$ The nilindex is 3 ; it corresponds to the rigid algebra in $\mathcal{N}^{3}$, generated by the basis $\left(X, X^{2}, X^{3}\right)$. It gives the irreducible component $C_{1}$.

$2^{9}$ ) The nilindex is 2 ; there is the algebra $A_{2}^{3}$ of the family (definition 2.1) which determines the irreducible component $C_{2}$.

$\left.3^{\circ}\right)$ and $\left.4^{9}\right)$ The nilindex is 1 . The case $\left(3^{\frac{9}{}}\right)$ give the skew-symmetric algebra generated by the basis $(X, Y, X \cdot Y)$. It's easily perturbed in algebra of larger nilindex, if we take $X^{2}=\varepsilon X \cdot Y$ with $\varepsilon \approx 0$. The case $\left(4^{\circ}\right)$ gives an isomorphic algebra to the previous one.

Remark. The minoration given before is optimal in this dimension.

VARIETY $\mathfrak{X}^{4}$

Proposition. The variety $\mathcal{X}^{4}$ has 4 irreducible components.

Proof. We discuss each occuring nilindex separately:

- Nilindex 4: There is the rigid algebra, generated by the basis $\left(X, X^{2}, X^{3}, X^{4}\right)$. It defines an irreducible component $C_{1}$. 
- Nilindex 3: There is the irreducible component given by the algebra $A_{3}^{4}$ (definition 2.1). It defines the irreducible component $C_{2}$.

- Nilindex 2: By the lemma, we have two possibilities for the matrix of $\mathrm{L}_{\mathrm{x}}$.

$$
\text { a) }\left(\begin{array}{llll}
0 & 0 & 0 & 0 \\
1 & 0 & 0 & 0 \\
0 & 0 & 0 & 0 \\
0 & 0 & 1 & 0
\end{array}\right) \quad \text { b) }\left(\begin{array}{llll}
0 & 0 & 0 & 0 \\
1 & 0 & 0 & 0 \\
0 & 0 & 0 & 0 \\
0 & 0 & 0 & 0
\end{array}\right)
$$

a) There is the algebra law $\mu_{1}$ generated by the basis $\left(X, X^{2}, Y, X \cdot Y\right)$. In this basis, the products which are not given directly by the basis are:

$$
\mu_{1}: Y \cdot X=\alpha X^{2}+\beta X \cdot Y \text { and } Y^{2}=\gamma X^{2}+\delta X \cdot Y .
$$

b) i) If the basis is $\left(X, X^{2}, Y, Y \cdot X\right)$ then there is the law $\mu_{2}$ where $Y^{2}=\alpha X^{2}+\beta Y \cdot X$. $\mathrm{Y} \cdot \mathrm{X}=\alpha \mathrm{X}^{2}$.

ii) If the basis is $\left(X, X^{2}, Y, Y^{2}\right)$ then there is the law $\mu_{3}$ where

iii) If the basis is $\left(X, X^{2}, Y, Z\right)$ then there is the law $\mu_{4}$ of $A_{2}^{4}$.

The law $\mu_{2}$ and $\mu_{3}$ belongs to the family $\mu_{1}$ with $\alpha=0$ and $\beta=\infty$ for $\mu_{2}$ and $\alpha=\beta=0$ and $\delta=\infty$ for $\mu_{3}$.

The law $\mu_{4}$ is not in the family $\mu_{1}$. And these algebras cannot be perturbed in algebras of larger nilindex. Therefore, $\mu_{4}$ and $\mu_{1}$ correspond to the irreducible components $\mathrm{C}_{3}$ and $\mathrm{C}_{4}$.

- Nilindex 1: the algebras obtained are contractions of algebras of larger nilindex. 


\section{VARIETY $\mathfrak{N}^{5}$}

Proposition. The variety $\mathcal{N}^{5}$ has 13 irreducible components.

Proof. We investigate the different values of the nilindex using the method described in the beginning.

Nilindex 5. There is, up to isomorphism, the rigid algebra generated by the basis $\left\{X, X^{2}, X^{3}, X^{4}, X^{5}\right\}$ and which define the irreducible component $\mathrm{C}_{1}$.

Nilindex 4 . Every law of $\mathfrak{N}^{5}$ with nilindex 4 is isomorphic, up to isomorphism, to one of the following laws, where $\left(X, X^{2}, X^{3}, X^{4}, Y\right)$ is a basis:

a) $X^{i} \cdot X^{j}=X^{i+j}$ if $i+j \leq 4 \quad i \leq i, j \leq 3$.

b) $X^{i} \cdot X^{j}=X^{i+j}$ if $i+j \leq 4 \quad 1 \leq i, j \leq 3 ; Y \cdot X=X^{4}$.

c) $X^{i} \cdot X^{j}=X^{i+j}$ if $i+j \leq 4 \quad 1 \leq i, j \leq 3 ; Y \cdot X=X^{4} ; Y \cdot Y=\alpha X^{4}$.

The laws (a) and (b) would be perturbed in an element of the family (c) which cannot be perturbed in the rigid law of nilindex 5 the (proposition 2.1). It defines the irreducible component $\mathrm{C}_{2}$.

Nilindex 3. Following the lemma, we have two possibilities of $\mathrm{L}_{\mathrm{x}}$.

$$
\text { a) }\left(\begin{array}{lllll}
0 & 0 & 0 & 0 & 0 \\
1 & 0 & 0 & 0 & 0 \\
0 & 1 & 0 & 0 & 0 \\
0 & 0 & 0 & 0 & 0 \\
0 & 0 & 0 & 1 & 0
\end{array}\right) \quad \text { b) }\left(\begin{array}{lllll}
0 & 0 & 0 & 0 & 0 \\
1 & 0 & 0 & 0 & 0 \\
0 & 1 & 0 & 0 & 0 \\
0 & 0 & 0 & 0 & 0 \\
0 & 0 & 0 & 0 & 0
\end{array}\right)
$$

a) In this case, let $\left(X, X^{2}, X^{3}, Y, X \cdot Y\right)$ be a basis, we have the reducible (up to isomorphism) family given by:

$X^{i} \cdot X^{j}=X^{i+j}$ if $i+j \leq 3 \quad 1 \leq i, j \leq 2 ; Y \cdot X=\alpha X^{2}+\beta X^{3}+\gamma X \cdot Y ; Y \cdot X^{2}=(\alpha+\gamma \alpha) X^{3} ;$ $X \cdot Y \cdot X=\alpha X^{3}$

$Y \cdot Y=\lambda X^{2}+\rho X^{3}+v X \cdot Y ; Y \cdot X \cdot Y=\gamma \lambda X^{3} ; X \cdot Y^{2}=\lambda X^{3}$. 
and the relations:

$$
\alpha^{2}+\gamma \alpha^{2}+\gamma^{2} \lambda-\lambda-v \alpha=0 \text { and } \lambda(\alpha+\alpha \gamma+v \gamma-v)=0 .
$$

-If $\lambda=0$, the first equation becomes $\alpha(\alpha+\gamma \alpha-v)=0$. For $\alpha=0$ and $v=\alpha+\gamma \alpha$ there are laws with the same orbit dimension as the law (c) of nilindex 4.

Then $\alpha=0$ corresponds to an irreducible component $C_{3}$ and $v=\alpha+\gamma \alpha$ to the component $\mathrm{C}_{4}$.

- If $\lambda \neq 0$, then $\lambda=1$,

$$
\alpha=\sqrt{\left(1-\gamma^{2}\right) / \gamma} \text { and } v=\sqrt{\left(1-\gamma^{2}\right) / \gamma} \cdot(1+\gamma) /(1-\gamma)
$$

defines the irreducible component $C_{5} .(\gamma=0$ is impossible and $\gamma=1$ is in the previous family where $\alpha=0$ and $v=\infty$ ).

b) In this case, there are two possibilities:

i) let $\left(X, X^{2}, X^{3}, Y, Z\right)$ be a basis, we have the family:

$X^{i} \cdot X^{j}=X^{i+j}$ if $i+j \leq 3 \quad 1 \leq i, j \leq 2 ; Y \cdot X=\alpha X^{3} ; Y \cdot Y=\lambda X^{2} ; Y \cdot Z=\gamma X^{3} ; Z \cdot X=\beta X^{3}$; $Z \cdot Y=p X^{3} ; Z \cdot Z=v X^{3}$. It defines the irreducible component $C_{6}$.

ii) let $\left(X, X^{2}, X^{3}, Y, Y^{2}\right)$ be a basis, we have the family:

$X^{i} \cdot X^{j}=X^{i+j} \quad$ if $i+j \leq 3 \quad 1 \leq i, j \leq 2 ; \quad Y \cdot Y=Y^{2} ; \quad Y \cdot X=\alpha X^{3} ; \quad Y \cdot Y^{2}=Y^{2} \cdot Y=a X^{3}$, $\mathrm{a}=0,1$.

When $a=1$, the corresponding algebra defines the irreducible component $\mathrm{C}_{7}$.

Nilindex 2. Following the lemma, we have 3 possibilities of $\mathrm{L}_{\mathrm{x}}$. 
a) $\left.\left.\left(\begin{array}{lllll}0 & 0 & 0 & 0 & 0 \\ 1 & 0 & 0 & 0 & 0 \\ 0 & 0 & 0 & 0 & 0 \\ 0 & 0 & 1 & 0 & 0 \\ 0 & 0 & 0 & 1 & 0\end{array}\right) \quad b\right)\left(\begin{array}{lllll}0 & 0 & 0 & 0 & 0 \\ 1 & 0 & 0 & 0 & 0 \\ 0 & 0 & 0 & 0 & 0 \\ 0 & 0 & 1 & 0 & 0 \\ 0 & 0 & 0 & 0 & 0\end{array}\right) \quad c\right)\left(\begin{array}{lllll}0 & 0 & 0 & 0 & 0 \\ 1 & 0 & 0 & 0 & 0 \\ 0 & 0 & 0 & 0 & 0 \\ 0 & 0 & 0 & 0 & 0 \\ 0 & 0 & 0 & 0 & 0\end{array}\right)$

We shall only write these products which are not defined directly by the basis.

a) Let $\left(X, X^{2}, Y, X \cdot Y, X^{2} \cdot Y\right)$ be the basis. We get the following families:

$Y \cdot X=-\alpha(2 a+1) X^{2}+a X \cdot Y+\gamma X^{2} \cdot Y ; \quad Y \cdot X^{2}=a^{2} X^{2} \cdot Y ; \quad Y \cdot Y=-\alpha^{2} X^{2}+\alpha X \cdot Y+$ $\beta X^{2} . \quad Y ; \quad Y \cdot X \cdot Y=-\alpha(a+1) X^{2} \cdot Y ; \quad X \cdot Y \cdot X=a X^{2} \cdot Y ; \quad X \cdot Y \cdot Y=\alpha X^{2} \cdot Y ;$ where $a=(-1 \pm i \sqrt{3}) / 2$.

They define the irreducible components $\mathrm{C}_{8}$ and $\mathrm{C}_{9}$.

b) We have two cases:

$\left.1^{9}\right)$ If the basis is $\left(X, X^{2}, Y, X \cdot Y, Y \cdot X\right)$, the law is defined by; $Y \cdot Y=\alpha X \cdot Y+\beta Y \cdot X$. There is a perturbation of nilindex 3 , if we take $X^{3}=\varepsilon X \cdot Y$ where $\varepsilon \propto 0$.

$\left.2^{9}\right)$ If the basis is $\left(X, X^{2}, Y, X \cdot Y, Z\right)$, we get the following law:

i) $Y \cdot X=\alpha_{1} X^{2}+\alpha_{2} X \cdot Y ; \quad Y \cdot Y=\beta_{1} X^{2}+\beta_{2} X \cdot Y ; \quad Y \cdot Z=\gamma_{1} X^{2}+\gamma_{2} X \cdot Y ;$ $Z \cdot X=\delta_{1} X^{2}+\delta_{2} X \cdot Y ; Z \cdot Y=\rho_{1} X^{2}+\rho_{2} X \cdot Y ; Z \cdot Z=\eta_{1} X^{2}+\eta_{2} X \cdot Y$.

If the basis is $\left(X, X^{2}, Y, X \cdot Y, Y^{2}\right)$, we have:

ii) $Y \cdot X=\alpha X^{2}+\beta X \cdot Y$.

iii) $Y \cdot X=\alpha X^{2} ; X \cdot Y^{2}=X^{2} ; X \cdot Y \cdot Y=X^{2}$. $\mathrm{C}_{12}$.

Then the laws $\mathrm{i}$, ii, iii give the irreducible components $\mathrm{C}_{10}, \mathrm{C}_{11}$ and 
c) We have 3 cases:

i) If the basis is $\left(X, X^{2}, Y, Y^{2}, Z\right)$, we obtain the family defined by:

$Y \cdot Z=\beta Y^{2} ; Z \cdot X=\alpha X^{2} ; Z \cdot Y=\beta Y^{2} ; Z \cdot Z=\gamma X^{2}+\lambda Y^{2}$. It is in the family (b.2.i).

ii) If the basis is $\left(\mathrm{X}, \mathrm{X}^{2}, \mathrm{Y}, \mathrm{Z}, \mathrm{Y} \cdot \mathrm{Z}\right)$, we obtain the familiy defined by:

$\mathrm{Y} \cdot \mathrm{X}=\alpha \mathrm{X}^{2} ; \mathrm{Y} \cdot \mathrm{Y}=\gamma \mathrm{X}^{2} ; \mathrm{Z} \cdot \mathrm{X}=\beta \mathrm{X}^{2} ; \mathrm{Z} \cdot \mathrm{Y}=\rho \mathrm{X}^{2}+\lambda \mathrm{Y}^{2}$. It is also in (b.2.j).

iii) If the basis is $\left(X, X^{2}, Y, Z, V\right)$, we obtain the family defined by:

$\mathrm{Y} \cdot \mathrm{X}=\beta \mathrm{X}^{2} ; \mathrm{Z} \cdot \mathrm{X}=\alpha \mathrm{X}^{2} ; \mathrm{V} \cdot \mathrm{X}=\gamma \mathrm{X}^{2} ; \mathrm{Y} \cdot \mathrm{Y}=\mathrm{aX} \mathrm{X}^{2} ; \mathrm{Y} \cdot \mathrm{Z}=\mathrm{bX} \mathrm{X}^{2} ; \mathrm{Y} \cdot \mathrm{V}=\mathrm{cX} \mathrm{X}^{2} ;$ $\mathrm{Z} \cdot \mathrm{Y}=\mathrm{eX}^{2} ; \mathrm{Z} \cdot \mathrm{Z}=\mathrm{gX}^{2} ; \mathrm{Z} \cdot \mathrm{V}=\mathrm{h} X^{2} ; \mathrm{V} \cdot \mathrm{Y}=\mathrm{mX} X^{2} ; \mathrm{V} \cdot \mathrm{Z}=\mathrm{pX} X^{2} ; \mathrm{V} \cdot \mathrm{V}=\mathrm{qX} X^{2}$.

It corresponds to the irreducible component $\mathrm{C}_{13}$.

Nilindex 1. Following the lemma, we have 3 possibilities of $\mathrm{L}_{\mathrm{x}}$.
a) $\left(\begin{array}{lllll}0 & 0 & 0 & 0 & 0 \\ 0 & 0 & 0 & 0 & 0 \\ 0 & 1 & 0 & 0 & 0 \\ 0 & 0 & 0 & 0 & 0 \\ 0 & 0 & 0 & 1 & 0\end{array}\right)$
b) $\left(\begin{array}{lllll}0 & 0 & 0 & 0 & 0 \\ 0 & 0 & 0 & 0 & 0 \\ 0 & 1 & 0 & 0 & 0 \\ 0 & 0 & 0 & 0 & 0 \\ 0 & 0 & 0 & 0 & 0\end{array}\right)$
c) null-matrix

a) Let $(X, Y, X \cdot Y, Z, X \cdot Z)$ be the basis, we have the following laws:

i) $Y \cdot X=-X \cdot Y ; Z \cdot X=-X \cdot Z ; Y \cdot Z=-Z \cdot Y=X \cdot Z$.

ii) $Y \cdot X=-X \cdot Y ; Z \cdot X=+X \cdot Z$.

The second law can be perturbed in the first. The first has a perturbation of nilindex 2 if we take in (i) $X \cdot X=\varepsilon X \cdot Y$ where $\varepsilon \sim 0$. 
b) Let $(\mathrm{X}, \mathrm{Y}, \mathrm{X} \cdot \mathrm{Y}, \mathrm{Z}, \mathrm{V})$ be the basis, we have the laws:

i) $Y \cdot X=-X \cdot Y$; $Z \cdot V=-V \cdot Z=X \cdot Y$. And ii) $Y \cdot X=-X \cdot Y$.

If $X \cdot X=\varepsilon X \cdot Y$, where $\varepsilon \approx 0$, we get a perturbation of (i) of nilindex 2. Hence (i) is a contraction of (ii).

c) We obtain in this case the null law.

These laws would be perturbed in laws of nilindex larger than one.

\section{RIGIDITY OF SIX-DIMENSIONAL NILPOTENT UNITARY ALGEBRAS}

Let $\mathrm{Alg}_{6}$ be the set of six-dimensional unitary associative algebras. A nilpotent algebra of $\mathrm{Alg}_{6}$ is an element of the direct product $\mathcal{N} \times \mathbb{C}$. An algebra is rigid if every perturbation is isomorphic to this algebra. The rigid algebras of $\mathrm{Alg}_{\mathrm{n}}$ are not nilpotent for $\mathrm{n} \leq 5$ (see [Ga], [Ma]). Since the variety $\mathcal{N}^{\delta}$ is described above, we will study the rigidity of these algebras viewed in $\mathrm{Alg}_{6}$.

Theorem. The nilpotent algebras of Alg are not rigid.

Proof. We examine the algebras of $\mathcal{N}^{5}$ following the nilindex.

Nilindex 5: The rigid algebra in $\mathcal{N}^{5}$ is not rigid in $\mathrm{Alg}_{6}$ (by adding the unit). In fact, we take $X^{i} \cdot X^{j}=\varepsilon X^{5}$ if $i+j=6$, where $\varepsilon \approx 0$. The perturbation is not nilpotent.

Nilindex 4: The law (c) is a perturbation of (a) and (b). And $\alpha$ is a perturbation parameter (deformation parameter) of the family (c).

\section{Nilindex 3:}

a) We have $\alpha$ and $\lambda$ which are perturbation parameter. The 2coboundary $\delta$ defined by $\delta f\left(X, Y^{2}\right)=\alpha X^{3}$ and $\delta f(Y, X \cdot Y)=\lambda X^{3}$ gives a non trivial perturbation of $\mu_{0}, \mu_{0}+\varepsilon \delta$ f where $\varepsilon \approx 0$. 
b) In (i) and (ii) $\alpha$ is a perturbation parameter.

Nilindex 2:

a) Here $\alpha$ is a perturbation parameter.

b.1) The laws would be perturbed in an algebra of nilindex 3 , hence they are not rigid.

b.2) For the laws (i) $\eta_{2}$ is a perturbation parameter and for the laws (ii) and (iii) we have $\alpha$ as a perturbation parameter.

c) The laws (i) and (ii) belongs to the family (b.2.i.). But in (iii) if $a \neq 0$ then $\alpha$ is a perturbaion parameter.

Nilindex I: All the laws would be perturbed in laws of larger nilindex, hence they are not rigid.

Since we get only families with perturbation parameters or algebras having a perturbation with different nilindex, there are no rigid nilpotent algebras in $\mathrm{Alg}_{6}$.

This result will be used in a forthcoming paper for classifying the rigid algebras of $\mathrm{Alg}_{6}$.

\section{References}

[A] ANCOCHEA-BERMUDEZ, J.M. Sur la classification des algèbres de Lie rigides. Thèse, Mulhouse, (1985).

[G] GOZE, M. Etude locales de la variété des algèbres de Lie. Thèse d'Etat, Mulhouse, (1982).

[G.M] GOZE, M. and MAKHLOUF, A. On the rigid complex associative algebras. Communications in Algebra, 18 (12), $4031-4046$ (1990).

[Ga] GABRIEL, P. Finite representation type is open. Proceedings of ICRAI, Ottawa 1974, LNM № 488, Springer-Verlag (1975). 
[H] HAPPEL, D. Deformations of five-dimensional algebras with unit. Ring Theory, Lect. notes in pure and appl. Math. (1978).

[Ma] MAZZOLA, G. The algebraic and geometric classification of associative algebras of dimension five. Manuscripta math. 27, (1979).

Université de Haute Alsace

Recibido: 3 de marzo de 1992

Faculté des Sciences et Techniques

32, rue du Grillenbreit

F-68100 Colmar

FRANCE 